\title{
Magnetization Reversal of Exchange-biased Bilayers and Trilayers Probed using Front and Back LT-MOKE
}

\author{
Ki-Yeon Kim ${ }^{1}$, Ji-Wan Kim ${ }^{2}$, Hyeok-Cheol Choi ${ }^{3}$, Chun-Yeol You ${ }^{3}$, Sung-Chul Shin ${ }^{2}$, and Jeong-Soo Lee ${ }^{1}$ \\ ${ }^{1}$ Neutron Science Division, Korea Atomic Energy Research Institute, Daejeon 305-353, Korea \\ ${ }^{2}$ Department of Physics, Korea Advanced Institute of Science and Technology, Daejeon 305-701, Korea \\ ${ }^{3}$ Department of Physics, Inha University, Incheon 402-751, Korea
}

(Received 4 December 2008, Received in final form 2 February 2009, Accepted 4 February 2009)

\begin{abstract}
Magneto-optical Kerr effect (MOKE) magnetometry was used to investigate magnetization reversal dynamics in 30-nm NiFe/15-nm FeMn, 15-nm FeMn/30-nm CoFe bilayers, and 30-nm NiFe/(2,10)-nm FeMn/30-nm CoFe trilayers. The in-plane magnetization components of each ferromagnetic layer, both parallel and perpendicular to the applied field, were separately determined by measuring the longitudinal and transverse MOKE hysteresis loops from both the front and back sides of the film for an oblique incident s-polarized beam. The magnetization of the $\mathrm{FeMn} / \mathrm{CoFe}$ bilayer was reversed abruptly and symmetrically through nucleation and domain wall propagation, while that of the $\mathrm{NiFe} / \mathrm{FeMn}$ bilayer was reversed asymmetrically with a dominant rotation. In the $\mathrm{NiFe} / \mathrm{FeMn} / \mathrm{CoFe}$ trilayers, the magnetic reversal of the two ferromagnetic layers proceeded via nucleation and domain wall propagation for $2-\mathrm{nm}$ FeMn, but via asymmetric rotation for 10-nm FeMn. The exchange-biased ferromagnetic layers showed the magnetization reversal along the same path in the film plane for the decreasing and increasing field branches from transverse MOKE hysteresis loops, which can be qualitatively explained by the theoretical model of the exchange-biased ferromagnetic/antiferromagnetic systems.
\end{abstract}

Keywords : exchange bias, asymmetric magnetization reversal, MOKE

\section{Introduction}

Vectorial determination of magnetization in magnetic thin films and multilayers can be the most direct solution for a three-dimensional magnetic configuration and also facilitates the study of magnetization reversal dynamics. Magnetic hysteresis $(M-H)$ loops from a vibrating sample magnetometer (VSM), a superconducting quantum interference device (Ed- the abbreviation is unnecessary as it is not used anywhere else in the paper), and a magnetooptic Kerr effect (MOKE) magnetometer usually provide a single magnetization component, parallel $\left(M_{\|}\right)$or perpendicular $\left(M_{\perp}\right)$ to an applied field $H$. Meanwhile, it is possible to measure both the $M_{\|}-H$ and $M_{\perp}-H$ loops from a longitudinal MOKE (L-MOKE) measurement by rotating both one sample and one magnet by 90 degrees and fixing the others for an oblique incident $s$ - or $p$ - polarized beam [1-3]. Moreover, because a direct comparison between the $M_{\|}-H$ and $M_{\perp}-H$ loops is possible in this mea-

*Corresponding author: Tel: +82-42-868-4647

Fax: +82-42-868-4629, e-mail: kykim3060@kaeri.re.kr surement geometry and the $M_{\perp}-H$ loop reveals the chirality, these allow us to determine the relative magnitude and direction of magnetization during reversal in a magnetic layer $[2,4]$.

Exchange bias is established after field-cooling a magnetic heterostructure containing a ferromagnet $(\mathrm{F}) /$ antiferromagnet (AF) interface across the AF blocking temperature or depositing it in the presence of an in situ magnetic field $[5,6]$. The exchange coupling across the F/AF interface leads to the displacement of a macroscopic $M-H$ loop of the $\mathrm{F}$ layer along the field axis away from the origin and accompanies its enhanced coercivity. The amount of displacement is called the exchange bias field $H_{E}$.

Various measurement techniques such as VSM, anisotropic magnetoresistance, polarized neutron reflectivity, soft $\mathrm{x}$-ray scattering, and MOKE have revealed the asymmetric form of the magnetization reversal of the exchangebiased $\mathrm{F}$ layer [3, 7-16]. The magnetization reversal takes place via magnetization rotation or via nucleation and domain wall propagation along the same or different sides of an applied field during the decreasing (from positive to negative saturation) and increasing (from negative to 
positive saturation) field branches of the $M-H$ loop. For example, Liu and Adenwalla proposed using the StonerWohlfarth model where the magnetization reversal in the exchange-biased F/AF bilayer strongly depends on the orientation of the applied field and the competition between the uniaxial and unidirectional anisotropies [3]. When the unidirectional anisotropy is comparable to or stronger than the uniaxial anisotropy, F spins always rotate along the same path for the decreasing and increasing branches of the $M-H$ loop. Paul et al. found from specular polarized neutron reflectivity that the magnetization reversal modes are affected by the direction of the applied field with respect to the cooling field direction in the exchange-biased polycrystalline $\mathrm{IrMn} / \mathrm{CoFe}$ bilayers. They claimed that the relative strength of the uniaxial and unidirectional anisotropies is responsible for the different magnetization reversals [15]. The origin of the experimentally observed asymmetry has been addressed as a higher magnetic anisotropy [7], an irreversible change of the AF domain state [8], a partial domain wall parallel to the interface in an F or AF layer [9], an anisotropy dispersion in an $\mathrm{F}$ or $\mathrm{AF}$ layer [11], the competition between anisotropies [3, 13, 15], the angle between an applied field direction and a cooling field direction [3, 14, 17]. Although extensive research has been conducted experimentally and theoretically, the fundamental theory explaining all the features of the magnetization reversal in many F/AF systems remains unknown and controversial due to the inherently complex F/AF spin structure and the difficulty in directly probing the spin configuration and crystallographic structure at an F/AF interface [18].

In this report, we determined the magnitude and direction of the in-plane magnetization vector of $\mathrm{NiFe}$ and $\mathrm{CoFe}$ layers in the exchange-biased $\mathrm{NiFe} / \mathrm{FeMn} / \mathrm{CoFe}$ trilayers grown on an optically transparent substrate by measuring the longitudinal (L-MOKE) and transverse (TMOKE) MOKE hysteresis loops from both the film and substrate sides for an oblique incident $s$-polarized beam [1-3].

\section{Experiment}

Trilayers consisting of 30-nm NiFe(bottom, F)/FeMn (AF)/30-nm CoFe (top, F) with different FeMn thicknesses up to $10 \mathrm{~nm}$ were grown on optically transparent SiN substrate at an ambient temperature using dc magnetron sputtering at an Ar working pressure of 1.5 mTorr. A 5-nm Ta underlayer to promote a FeMn(111) texture and a 5-nm Ta capping layer to prevent oxidation were incorporated. In addition, 30-nm NiFe/15-nm FeMn and 15-nm FeMn/30-nm CoFe bilayers and 30-nm NiFe and 30-nm CoFe single layers were prepared for comparison with the trilayers. The sample preparation has already been described elsewhere [19]. A magnetic field of 300 Oe was applied along the sample plane during deposition to induce the exchange anisotropy. Crystallographic structures such as the film thickness and the surface/interface roughness and growth texture were characterized by lowangle x-ray reflectivity and high-angle $\theta / 2 \theta$ x-ray diffraction. To measure the magnitude and direction of the inplane magnetization vector of each $F$ layer selectively, we used the MOKE setup shown in Fig. 1. We used the Ti: sapphire femtosecond laser system with a typical center wavelength of $780 \mathrm{~nm}$ and repetition rate of $82 \mathrm{MHz}$. The probe power of $100 \mu \mathrm{A}(\sim \mathrm{pJ} /$ pulse $)$ was too small to change the magnetic state of the sample. To enhance the signal to noise ratio, we used the lock-in technique. The chopper frequency was optimized to $420 \mathrm{~Hz}$ and its corresponding lock-in acquisition time was set to $100 \mathrm{~ms}$. Therefore, every data point, even in a single loop measurement, already averaged over about 40 lock-in signals. The $s$-polarized light beam with a wavelength of $800 \mathrm{~nm}$ was focused on the $2 \mathrm{~mm}$ sample with an incident angle of about $30^{\circ}$. The sample was placed between the poles of the horizontal and vertical electromagnets. Firstly, $s$ polarized light put a Lorentz force only on the magnetic component parallel to the horizontal applied field. This is the L-MOKE (Ed- this abbreviation has already been defined above) hysteresis loop. Then, by rotating the sample by 90 degrees and using a vertical electromagnet, only a magnetic component perpendicular to the applied field contributes to the MOKE hysteresis in this geometry. This is the T-MOKE hysteresis loop. To avoid a possible training effect in the exchange-biased system, we repeated every loop measurement at least 5 times and averaged those signals. To measure the LT-MOKE of each $\mathrm{F}$ layer selectively in our trilayers, we prepared samples on the transparent SiN substrate and optimized each layer thickness of $\mathrm{NiFe}, \mathrm{CoFe}$, and Ta by taking into account the typical 25 30 nm skin depth of the $800 \mathrm{~nm}$ laser beam. Therefore, using our MOKE setup, we could determine the magnitude and direction of the in-plane $\mathrm{F}$ magnetization vector in the exchange-biased $\mathrm{NiFe} / \mathrm{FeMn} /$ CoFe trilayers with layer selectivity.

\section{Results and Discussion}

Fig. 2 shows the LT-MOKE loops for both $\mathrm{CoFe}$ and $\mathrm{NiFe}$ single layers and from the front and back sides of both $\mathrm{NiFe} / \mathrm{FeMn}$ and $\mathrm{FeMn} / \mathrm{CoFe}$ bilayers. A non-vanishing T-MOKE was only observed in the front/back side of the $\mathrm{NiFe} / \mathrm{FeMn}$ bilayer, and not in the $\mathrm{FeMn} / \mathrm{CoFe}$ bi- 


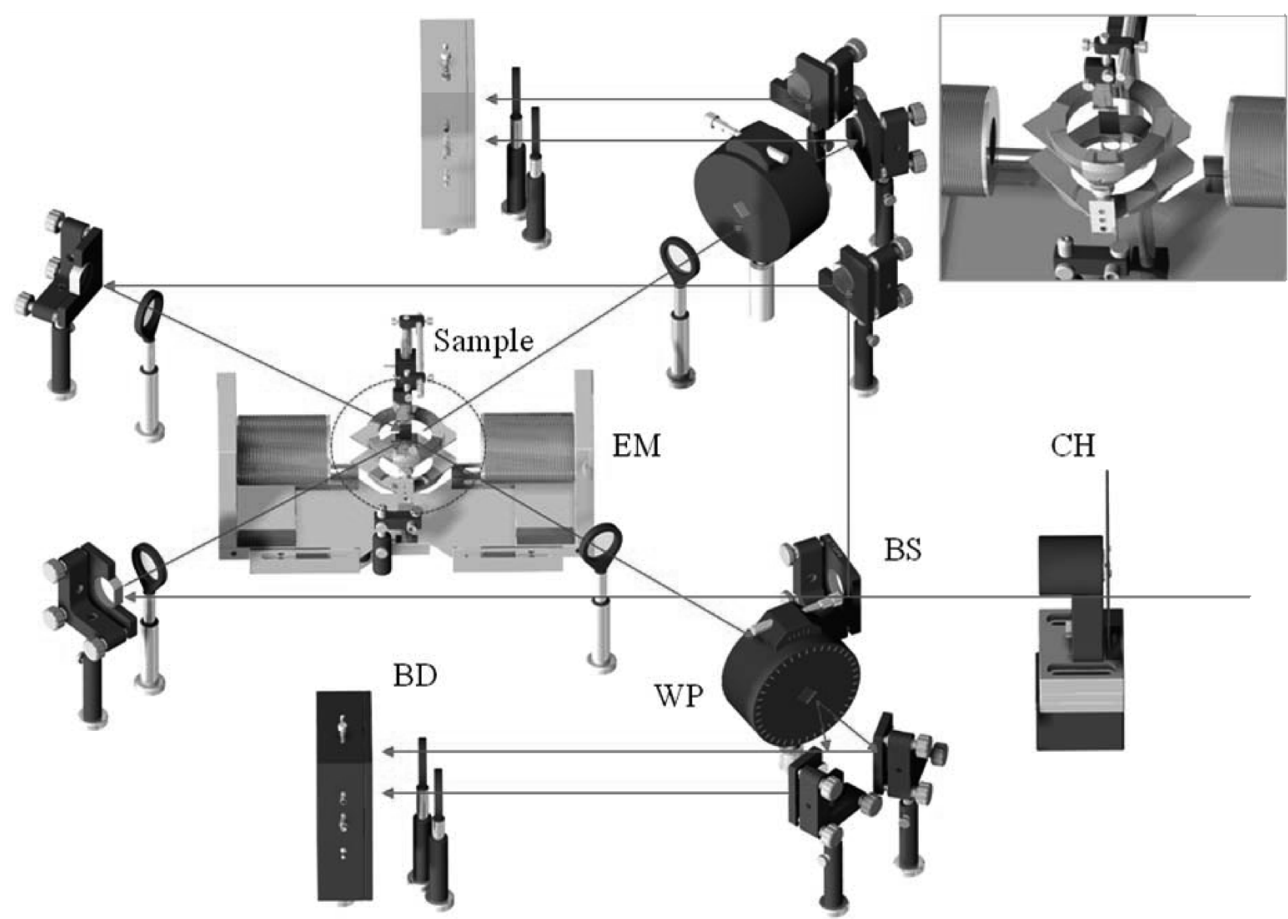

Fig. 1. Experimental configuration for the longitudinal and transverse MOKE measurements with which the longitudinal and transverse signals were measured using horizontally and vertically positioned, Helmholtz electromagnets, respectively. The red lines show the s-polarized probe beam path. The inset shows the magnified picture of a sample region. $\mathrm{CH}$ : chopper, BS: beam splitter, EM: electromagnet, WP: Wollaston prism, BD: balanced detector.

layer. This indicated that the reversal occurred dominantly through magnetization rotation in the exchange biased $\mathrm{NiFe} / \mathrm{FeMn}$ bilayer and through nucleation and domain wall propagation in the $\mathrm{FeMn} / \mathrm{CoFe}$ bilayer [4]. Although only L-MOKE is displayed for the single $\mathrm{CoFe}$ and $\mathrm{NiFe}$ layers in Fig. 2(a) and (d), respectively, T-MOKE must have been absent because it was not observed for either the $\mathrm{NiFe} / \mathrm{FeMn}$ or $\mathrm{FeMn} / \mathrm{CoFe}$ layer with a FeMn thickness of less than 5-nm, as shown in Fig. 3(a) and 3(b). For the exchange-biased $\mathrm{FeMn} / \mathrm{CoFe}$ bilayer, $H_{E}$ was almost zero and the coercivity $H_{C}$ was increased compared with the corresponding single layer for the front and back side, respectively. That is the case expected for weak $\mathrm{AF}$ anisotropy [5]. The coercivity of the front side in Fig. 2(b) is smaller than that of the back side in Fig. 2(c), indicating that the closer the $\mathrm{CoFe}$ spins are located to the $\mathrm{FeMn} / \mathrm{CoFe}$ interface, the stronger they are exchangecoupled with the FeMn spins. It seems that the magnetization reversal occurs via nucleation and domain wall motion symmetrically, because the normalized Kerr intensities change abruptly around the left and right coercivity. For the exchange-biased $\mathrm{NiFe}$ layer, on the other hand, $H_{E}$ was about -20 Gauss and $H_{C}$ was about -11.1 Oe and -9.4 Oe for the front and back sides, respectively. TMOKE loops for the front and back sides of the NiFe/
FeMn bilayer had the same sign during a complete hysteresis loop, implying that the magnetization doesn't rotate a full 360 degrees by itself but rotates coherently within a half circle of 180 degrees $[5,13]$. We determined the uniaxial and unidirectional anisotropies separately from the in-plane angular dependence of the resonance field for the $\mathrm{NiFe}$ and $\mathrm{CoFe}$ layers in the $\mathrm{NiFe} / \mathrm{FeMn} /$ $\mathrm{CoFe}$ trilayers using ferromagnetic resonance experiments, respectively [20]. The unidirectional anisotropy was stronger than the uniaxial one in both the $\mathrm{NiFe} / \mathrm{FeMn}$ and $\mathrm{FeMn} / \mathrm{CoFe}$ layers. The T-MOKE loops for the NiFe/ FeMn bilayer were in accordance with Liu and Adenwalla's model concerning the magnetization reversal in an exchange biased F/AF bilayer for a weak uniaxial anisotropy compared with a unidirectional anisotropy [3]. In addition to the sign, the shape of the T-MOKE loops is also of interest. Coercivity in the front side T-MOKE is not the same as that in the back side, and the amplitudes at the left and right coercivities are asymmetric, indicating that the amount of $\mathrm{F}$ spins differ from each other during magnetization rotation between the decreasing and increasing field branches of the T-MOKE loops from the front and back sides of the $\mathrm{NiFe} / \mathrm{FeMn}$ bilayer. This asymmetric magnetization reversal has been experimentally and theoretically attributed to typical phenomena in a number 


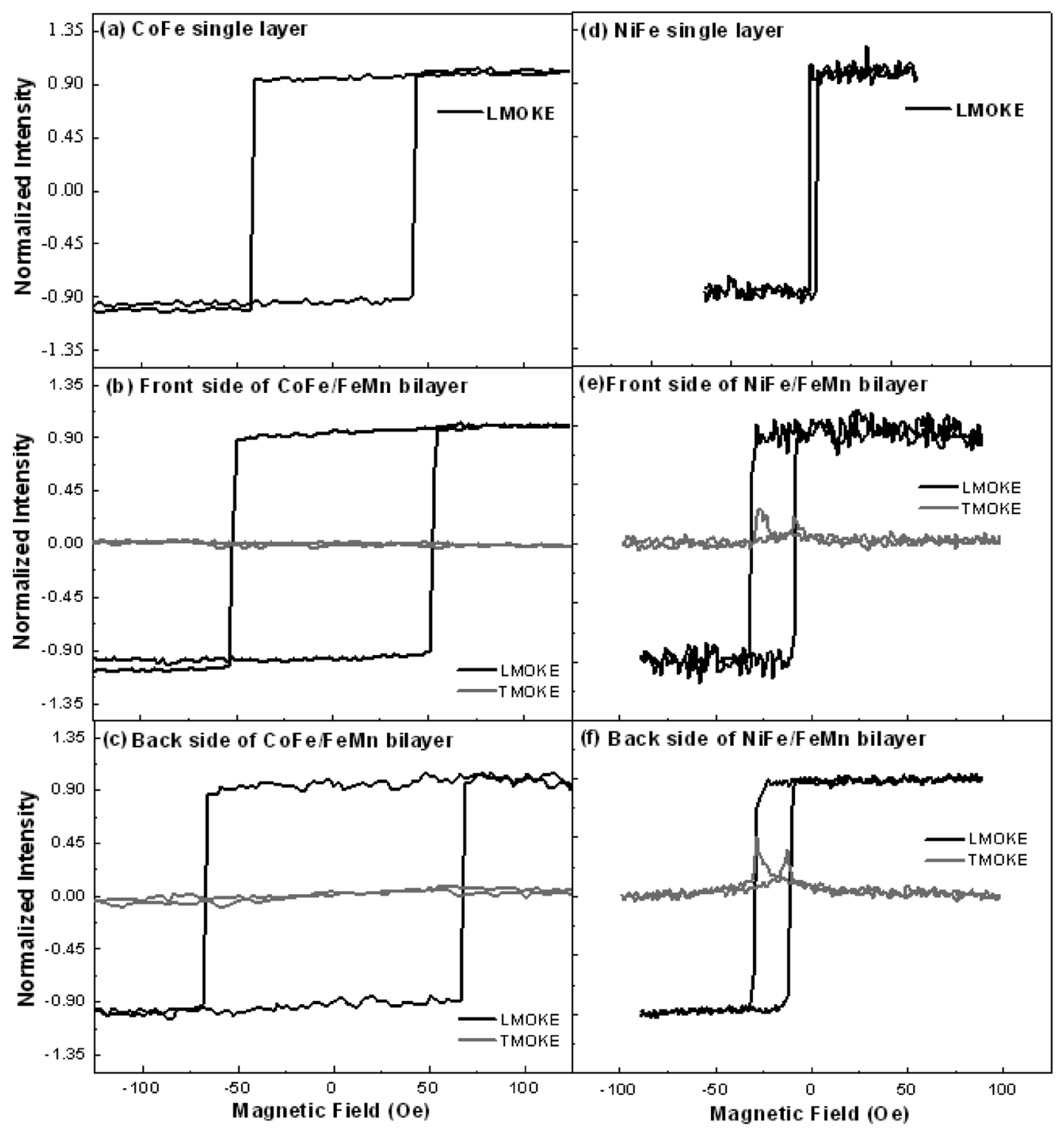

Fig. 2. L-(black line) MOKE loops for (a) the single 30-nm CoFe layer and (d) the single 30-nm NiFe layer, T-(red line) MOKE loops from both (b) front and (c) back sides of the 30-nm CoFe/15-nm FeMn bilayer, and LT-MOKE loops from both (e) front and (f) back sides of the 30-nm NiFe/15-nm FeMn bilayer grown on optically transparent SiN substrates (Ed- there is no respective comparison here).

of exchange-biased F/AF systems [3, 7-16]. The asymmetric T-MOKE loop for a NiFe/FeMn bilayer seems to originate from the competition between the uniaxial and unidirectional anisotropies $[3,13,15]$.

The LT-MOKE loops for the 30-nm NiFe/ $t_{A F}-\mathrm{nm}$ FeMn/ $30-\mathrm{nm}$ CoFe trilayers $\left(t_{A F}=2,10\right)$ were measured and compared to those of the single layer and bilayer, as shown in Fig. 3. For the 2-nm FeMn layer in Fig. 3(a) and 3(b), no $H_{E}$ was observed for the $\mathrm{NiFe} / \mathrm{FeMn}$ and $\mathrm{FeMn} / \mathrm{CoFe}$ interfaces because the 2-nm FeMn thickness was smaller than the critical FeMn thickness $(\sim 5 \mathrm{~nm})$ for the observation of $H_{E}$. However, $H_{C}$ was greatly increased compared to the corresponding $\mathrm{NiFe}$ single layer due to local exchange coupling at each F/AF interface. Only the L-MOKE loop was observed, indicating that the magnetization reversal occurs through nucleation and domain wall motion for both the $\mathrm{NiFe} / \mathrm{FeMn}$ and $\mathrm{FeMn} / \mathrm{CoFe}$ interfaces. This is similar to the case for the single $\mathrm{F}$ layer. In addition, the coercivities between the $\mathrm{NiFe} / \mathrm{FeMn}$ and $\mathrm{FeMn} / \mathrm{CoFe}$ interfaces were very similar, indicating that two $\mathrm{F}$ layers were exchange-coupled to each other across the 2-nm FeMn layer. For the 10-nm FeMn layer in Fig. 3(c) and 3(d), on the other hand, the LT-MOKE loops for each interface changed dramatically due to the 


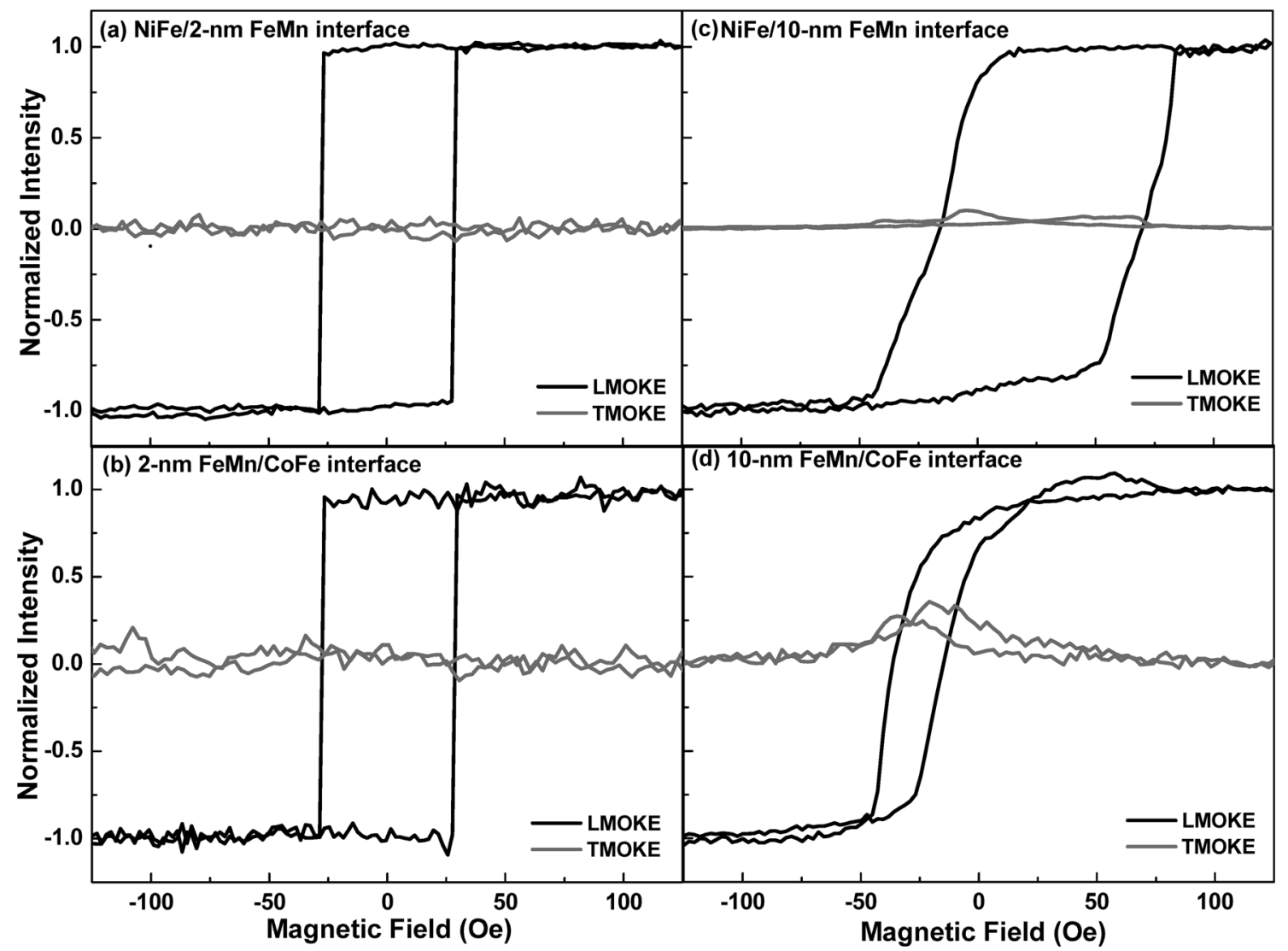

Fig. 3. L-(black line) and T-(red line) MOKE loops from both the NiFe/FeMn (a, c) and FeMn/CoFe (b, d) interfaces in the 30-nm $\mathrm{NiFe}$ (bottom) $/ t_{A F}$-nm FeMn/30-nm CoFe(top) trilayers with $t_{A F}=2$ and 10.

interfacial exchange coupling between $\mathrm{F}$ and $\mathrm{AF}$ layers. These two layers were biased oppositely with respect to each other. The $\mathrm{NiFe} / \mathrm{FeMn}$ interface was negatively biased $\left(H_{E} \sim 27.5 \mathrm{Oe}\right)$ along the field axis, while the FeMn/CoFe one was positively biased $\left(H_{E} \sim-25.7 \mathrm{Oe}\right)$ along the field axis. This was attributed to the antiferromagnetic spin structure at both FeMn interfaces. In addition to $H_{E}$, the coercivity $H_{C}$ increased to 42.9 Oe for the $\mathrm{NiFe} / \mathrm{FeMn}$ interface but decreased to 10.6 Oe for the $\mathrm{FeMn} / \mathrm{CoFe}$ one, compared to $H_{C} \sim 28$ Oe for the 2-nm FeMn case in Fig. 3(a) and 3(b), respectively. The T-MOKE loops for the two F/AF interfaces revealed that the magnetization reversal occurred through rotation within a half cycle of 180 degrees and was asymmetric between the decreasing and increasing field branches of the MOKE loop. The magnetization reversal of the $\mathrm{FeMn} / \mathrm{CoFe}$ interface in the trilayers was markedly different from that of the FeMn/ CoFe bilayers. Although the FeMn layer thickness was not the same for the $\mathrm{FeMn} / \mathrm{CoFe} / \mathrm{NiFe}$ trilayers and the FeMn/CoFe bilayer, we believe that our experimental observations support the conclusion that the exchange coupling at the two F/AF interfaces considerably affected the magnetization reversal behavior across the FeMn layer in the $\mathrm{NiFe} / \mathrm{FeMn} / \mathrm{CoFe}$ trilayers.

\section{Conclusion}

We investigated the magnetization reversal behavior due to the exchange coupling between an F/AF interface in 30-nm (NiFe, CoFe)/15-nm FeMn bilayers, and 30-nm $\mathrm{NiFe} / t_{A F}-\mathrm{nm}$ FeMn/30-nm CoFe trilayers $\left(t_{A F}=2,10\right)$ grown on an optically transparent substrate using magnetron sputtering. The in-plane magnetization components of each F layer, both parallel to and perpendicular to the applied field, were determined separately by measuring the L-MOKE and T-MOKE hysteresis loops from both the front and back sides of the film for an oblique incident $s$-polarized beam. We observed the asymmetric magnetization reversal through rotation from a nonvanishing $\mathrm{T}-\mathrm{MOKE}$ in the $\mathrm{NiFe} / \mathrm{FeMn}$ bilayer, a depthdependent coercivity enhancement due to the exchange coupling, and a magnetization reversal through nucleation and domain wall motion in the $\mathrm{FeMn} / \mathrm{CoFe}$ bilayer (Edthere is no respective comparison here). In the NiFe/ 
$\mathrm{FeMn} / \mathrm{CoFe}$ trilayers, the magnetic reversal of the two $\mathrm{F}$ layers proceeded through nucleation and domain wall propagation for 2-nm FeMn, but through rotation for 10$\mathrm{nm}$ FeMn. This experimental observation supported our conclusion that the exchange coupling at the two F/AF interfaces in the $\mathrm{NiFe} / \mathrm{FeMn} / \mathrm{CoFe}$ trilayers considerably affected the magnetization reversal across the FeMn layer. In addition, the exchange-biased $F$ layers showed magnetization rotation within a half cycle of 180 degrees for the decreasing and increasing field branches from the TMOKE hysteresis loops, which can be explained with Liu and Adenwalla's model.

\section{Acknowledgment}

This work was supported by KOSEF (Nuclear R\&D program) and KICOS (Global Partnership Program, No. K20702020014) through a grant provided by the Korean government (MEST). CYY was supported by Nano R\&D program through the Korea Science and Engineering Foundation funded by the Ministry of Science \& Technology (2008-02553).

\section{References}

[1] Chun-Yeol You and Sung-Chul Shin, J. Appl. Phys. 84, 541 (1998).

[2] C. Daboo, J. A. C. Bland, R. J. Hicken, A. J. R. Ives, M. J. Baird, and M. J. Walker, Phys. Rev. B 47, 11852 (1993).

[3] Z. Y. Liu and S. Adenwalla, J. Appl. Phys. 93, 3422 (2003).

[4] Florin Radu, Andreas Westphalen, Katharina Theis-Brohl, and Hartmut Zabel, J. Phys.: Condens. Matter 18, L29 (2006).

[5] J. Nogues and I. K. Shuller, J. Magn. Magn. Mater. 192,
203 (1999).

[6] J. Bass, A. Sharma, Z. Wei, and M. Tsoi, J. Magnetics 13, 1 (2008).

[7] I. N. Krivorotov, C. Leighton, J. Nogues, Ivan K. Schuller, and E. Dan Dahlberg, Phys. Rev. B 65, 100402-1 (2002).

[8] F. Radu, M. Etzkorn, T. Schmitte, R. Siebrecht, A. Schreyer, K. Westerholt, and H. Zabel, J. Magn. Magn. Mater. 240, 251 (2002).

[9] Z.-P. Li, O. Petracic, R. Morales, J. Olamit, X. Batlle, K. Liu, and I. K. Schuller, Phy. Rev. Lett. 96, 217205-1 (2006).

[10] E. Girgis, R. D. Portugal, H. Loosvelt, M. J. Van Beal, I. Gordon, M. Malfait, K. Temst, and C. Van Haesendonck, L. H. A. Leunissen, and R. Jonckheere, Phys. Rev. Lett. 91, 187202-2 (2003).

[11] J. McCord, R. Schäfer, R. Mattheis, and K.-U. Barholz, J. Appl. Phys. 93, 5491 (2003).

[12] J. Eisenmenger, Z.-P. Li, W. A. A. Macedo, and I. K. Schuller, Phys. Rev. Lett. 94, 057203 (2005).

[13] J. Camarero, J. Sort, A. Hoffmann, J. M. García-Martín, B. Dieny, R. Miranda, and J. Nogués, Phys. Rev. Lett. 95, 057204-1 (2005).

[14] A. Tilmanns, S. Oertker, B. Beschoten, G. Güntherodt, C. Leighton, and I. K. Schuller, J. Nogués, Apl. Phys. Lett. 89, 202512 (2006).

[15] A. Paul, E. Kentzinger, U. Rücker, and T. Brückel, J. Phys.: Condens. Matter 18, L149 (2006).

[16] K.-S. Lee, S.-K. Kim, J. B. Kortright, K.-Y. Kim, and S.C. Shin, J. of Magnetics 10(1), 36 (2005).

[17] B. Beckermann, U. Nowak, and K. D. Usadel, Phys. Rev. Lett. 91, 187201-1 (2003).

[18] K.-Y. Kim, Y.-S. Hwang, J.-G. Park, N. Torikai, M. Takeda, S.-W. Han, and S.-C. Shin, Phys. Stat. Sol. (b) 244, 4499 (2007).

[19] K.-Y. Kim, H.-C. Choi, C.-Y. You, and J.-S. Lee, J. Magnetics 13, 97 (2008).

[20] K.-Y. Kim, H.-C. Choi, J.-H. Shim, D.-H. Kim, C.-Y. You, and J.-S. Lee, (submitted). 